studied at their dorsal surface. The ultrasound has objectified the presence of two signs: hyperechoic band over the superficial margin of the articular cartilage described as a double contour (DC) and tophaceous deposits at the joint cavity. Results: The mean age at onset was $54.7 \pm 12,6$ years, and the median diagnosis duration was 0 (0.3) years).

The results of the US examination are summarized in Table 1.

Table 1. Prevalence of ultrasonographic gout specific signs of hind-foot, mid-foot and Metatarsophalangeal joints in the studied population

\begin{tabular}{lcc}
\hline Joints $(\mathrm{N}=330)$ & Double contour $(\%)$ & Tophaceous deposits $(\%)$ \\
\hline Hind-foot & 8,8 & 10 \\
Talocrural $(\mathrm{N}=30)$ & 13,3 & 13,3 \\
Subtalar: & & \\
Lateral $(\mathrm{N}=30)$ & 3,3 & 10 \\
Medial $(\mathrm{N}=30)$ & 10 & 6,7 \\
Mid-foot & 14,4 & 32,2 \\
Talonavicular $(\mathrm{N}=30)$ & 13,3 & 13,3 \\
Intertarsal $(\mathrm{N}=30)$ & 6,7 & 43,3 \\
Tarsometatarsal $(\mathrm{N}=30)$ & 23,3 & 40 \\
MTP $(\mathrm{N}=150)$ & 11,3 & 18 \\
MTP 1 $(\mathrm{N}=30)$ & 33,3 & 56,7 \\
MTP 2 $(\mathrm{N}=30)$ & 13,3 & 13,3 \\
MTP 3 $(\mathrm{N}=30)$ & 6,7 & 3,3 \\
MTP 4 $(\mathrm{N}=30)$ & 0 & 6,7 \\
MTP 5 $(\mathrm{N}=30)$ & 3,3 & 10
\end{tabular}

Conclusions: This study showed a predilection for the gout specific ultrasound signs (DC and tophaceous deposits) in the tarsometatarsal and metatarsophalangeal joints, especially in the first MTP. The contribution of musculoskeletal ultrasound seems to be very interesting to objectify the presence of gout specific signs of the foot joints.

Disclosure of Interest: None declared

DOI: 10.1136/annrheumdis-2017-eular.2468

\section{AB0886 PHARMACOKINETICS, PHARMACODYNAMICS, AND TOLERABILITY OF VERINURAD (RDEA3170), A SELECTIVE URIC ACID REABSORPTION INHIBITOR, IN HEALTHY ADULT MALE SUBJECTS}

M. Gillen ${ }^{1}$, Z. Shen ${ }^{2}$, J.N. Miner ${ }^{2} .{ }^{1}$ AstraZeneca, Gaithersburg, MD; ${ }^{2}$ Ardea Biosciences, Inc., San Diego, CA, United States

Background: Verinurad (RDEA3170) is a selective uric acid reabsorption inhibitor in clinical development for the treatment of gout and asymptomatic hyperuricemia. Objectives: The aim of this study was to evaluate the pharmacokinetics, pharmacodynamics, and tolerability of verinurad following single and multiple doses in healthy adult males.

Methods: This was a Phase 1, randomized, double-blind, placebo-controlled, single- and multiple-ascending dose study. Panels of 8 male subjects (6 active, 2 placebo) received a single oral dose of verinurad or placebo in either a fasted ( $2 \mathrm{mg}, 5 \mathrm{mg}, 20 \mathrm{mg}, 40 \mathrm{mg}$ ) or fed ( $5 \mathrm{mg}, 20 \mathrm{mg}$ ) state and panels of 12 male subjects (9 active, 3 placebo) received ascending doses of once-daily verinurad (1 $\mathrm{mg}, 5 \mathrm{mg}$, and $10 \mathrm{mg}$ ) or placebo in a fasted state for up to 10 days. Verinurad was administered as an oral solution for 1 and $2 \mathrm{mg}$ doses and in tablet form for doses $>2 \mathrm{mg}$. Serial plasma/serum and urine samples were assayed for verinurad and uric acid at predetermined time points. Safety was assessed by adverse event (AE) reports, laboratory tests, vital signs, and electrocardiograms (ECGs).

Results: A total of 81 adult males aged 18-54 years enrolled and completed the study. Following single oral doses of verinurad, absorption was rapid and exposure (maximum plasma concentration $\left[\mathrm{C}_{\max }\right]$ and area under the plasma concentrationtime curve $[\mathrm{AUC}]$ ) increased in a dose-proportional manner up to the maximum dose tested; $\mathrm{C}_{\max }$ was achieved at $0.5-0.75$ hours post-dose in the fasted state, and was slightly delayed to 1.25 hours post-dose in the fed state. Food appeared to decrease AUC by about $23 \%$ and $\mathrm{C}_{\max }$ by about $50 \%$. Following multiple daily doses, there was modest accumulation of verinurad. Urinary excretion of verinurad accounted for approximately $2 \%$ of the administered dose, suggesting that renal excretion is a minor elimination pathway for unchanged verinurad. Reductions in

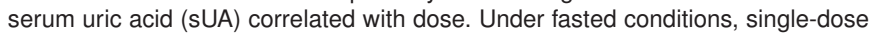
administration of verinurad $2,5,20$, or $40 \mathrm{mg}$ reduced sUA levels by $16 \%, 24 \%$, $48 \%$, and $62 \%$, respectively. Following multiple once-daily dosing for 10 days, verinurad reduced sUA levels by $22 \%, 44 \%$, and $61 \%$ for the 1,5 , and $10 \mathrm{mg}$ doses, respectively. A persistent pharmacologic effect ( $>15 \%$ fractional excretion of uric acid relative to baseline) was evident for at least 24 hours after dosing for verinurad doses of $2 \mathrm{mg}$ or above. Verinurad was well tolerated at all doses. No serious AEs, severe AEs, discontinuations due to AEs or clinically significant laboratory or ECG abnormalities were reported.

Conclusions: Single and multiple doses of verinurad were well tolerated, absorption was rapid and exposure was dose-proportional. Verinurad increased urinary uric acid elimination and resulted in sustained reductions in sUA. These data support further clinical evaluation of once-daily verinurad as a treatment for gout.

Acknowledgements: The authors thank Caroline Lee of Ardea Biosciences, Inc. for critical review of the abstract.
Disclosure of Interest: M. Gillen Employee of: AstraZeneca, Z. Shen Employee of: Ardea Biosciences, Inc., J. Miner Employee of: Ardea Biosciences, Inc. DOI: 10.1136/annrheumdis-2017-eular.5133

\section{AB0887 PHARMACOKINETICS, PHARMACODYNAMICS, AND TOLERABILITY OF VERINURAD (RDEA3170), A SELECTIVE URIC ACID REABSORPTION INHIBITOR, IN HEALTHY JAPANESE MALE SUBJECTS}

M. Gillen ${ }^{1}$, J.N. Miner ${ }^{2}$, S. Valdez ${ }^{2} .{ }^{1}$ AstraZeneca, Gaithersburg, MD; ${ }^{2}$ Ardea Biosciences, Inc., San Diego, CA, United States

Background: Chronic gout is a significant clinical problem in Asia, including Japan, where many patients remain suboptimally treated with currently available therapies. Verinurad (RDEA3170) is a selective uric acid reabsorption inhibitor in clinical development for the treatment of gout and asymptomatic hyperuricemia.

Objectives: The aim of this study was to evaluate the pharmacokinetics, pharmacodynamics, and tolerability of verinurad in healthy Japanese and nonAsian adult male subjects.

Methods: This was a Phase 1, randomized, single-blind, placebo-controlled study (NCT01872832). Panels of 8 Japanese male subjects were randomized in a 3:1 ratio to receive a modified-release formulation of oral verinurad $(2.5 \mathrm{mg}, 5 \mathrm{mg}$, $10 \mathrm{mg}, 15 \mathrm{mg}$ ) or placebo administered as a single dose in a fasted state and as multiple once-daily doses in a fed state for 7 days. A panel of 8 non-Asian male subjects received single and multiple doses of oral verinurad $(10 \mathrm{mg})$ or placebo. Serial plasma/serum and urine samples were assayed for verinurad and uric acid at predetermined time points. Safety was assessed by adverse event (AE) reports, laboratory tests, vital signs, and electrocardiograms (ECGs).

Results: Of 48 randomized subjects, 46 (Japanese: 39, non-Asian: 7) completed the study. Treatment groups were generally well balanced; however, mean body weight and body mass index were approximately $14 \%$ and $7 \%$ lower, respectively, in Japanese than non-Asian subjects. Following single- or multiple-oral doses of verinurad in Japanese subjects, exposure (maximum plasma concentration $\left[\mathrm{C}_{\max }\right]$ and area under the plasma concentration-time curve [AUC]) increased in a near dose-proportional manner under fasted or fed conditions. The time to $\mathrm{C}_{\max }$ ( $T_{\max }$ ) was approximately $1.25-2.0$ hours post-dose under fasted conditions. A moderate-fat meal delayed $\mathrm{T}_{\max }$ up to 5 hours post-dose and increased plasma verinurad exposures up to $109 \%$. Following once-daily multiple doses, there was modest accumulation of verinurad. $\mathrm{C}_{\max }$ and AUC were $38 \%$ and $23 \%$ higher, respectively, in Japanese versus non-Asian subjects, largely due to the difference in body weight. Mean reductions in serum uric acid following once-daily multiple dosing of verinurad $10 \mathrm{mg}$ were $62 \%$ and $58 \%$ at maximum reduction and $46 \%$ and $44 \%$ at 24 hours post-dose in Japanese and non-Asian subjects, respectively. Verinurad was well tolerated at all doses. One Japanese subject discontinued verinurad due to an $A E$ of urticaria that resolved after 11 days. No serious AEs, Grade 3 or $4 \mathrm{AEs}$, or clinically significant laboratory or ECG abnormalities were noted. Conclusions: Verinurad significantly lowered serum uric acid and was well tolerated in both healthy Japanese and non-Asian males, despite small differences in plasma pharmacokinetics. These data support further evaluation of once-daily verinurad as a treatment for hyperuricemia with or without gout in the Japanese population.

Acknowledgements: The authors thank Caroline Lee and Zancong Shen of Ardea Biosciences, Inc., for critical review of the abstract.

Disclosure of Interest: M. Gillen Employee of: AstraZeneca, J. Miner Employee of: Ardea Biosciences, Inc., S. Valdez Employee of: Ardea Biosciences, Inc. DOI: 10.1136/annrheumdis-2017-eular.5200

\section{AB0888 TENOFOVIR INDUCED OSTEOMALACIA: A PROFILE BASED ON THREE PATIENTS WITH LOW PHOSPHORUS AND NORMAL LEVELS OF VITAMIN D AND PARATHYROID HORMONE}

M. Lovy. Desert Oasis Healthcare, Palm Springs, California, United States

Background: Tenofovir can induce proximal renal tubular changes that result in varying expression of Fanconi syndrome ${ }^{1,2}$. Less commonly, osteomalacia related to hypophosphatemia ${ }^{2,3}$ can occur and has been documented with bone biopsy ${ }^{3}$. The clinical details of 28 reported cases of tenofovir induced osteomalacia, some of whom had vitamin D deficiency and secondary hyperparathyroidism, was recently summarized ${ }^{3}$.

Objectives: To describe the clinical presentation and course of three HIV patients with tenofovir induced hypophosphatemic osteomalacia and compare to cases previously reported.

Methods: The clinical, laboratory, and radiologic features of three HIV patients referred for evaluation of pain and osteoporosis were reviewed.

Results: All three patients were male, had diffuse pain, suffered multiple clinical fractures, and were on combination long and short acting opioids at the time of presentation. Two were in wheelchairs and two had neuropathy.

All patients had hypogonadism and proteinuria and case 1 had glycosuria. All patients had normal $25-\mathrm{OH}$ vitamin D, vitamin B12, $\mathrm{PTH}$, serum protein electrophoresis, magnesium, CBC, calcium, CPK, sedimentation rate, TSH. Case 1 had fractures of the hip, sacrum, and humerus; case 2 hip and ribs; and case 3 ribs, pelvis and knee. The technetium bone scan showed a similar pattern of increased uptake in multiple ribs, calcaneus, metatarsal bones, knees, and 